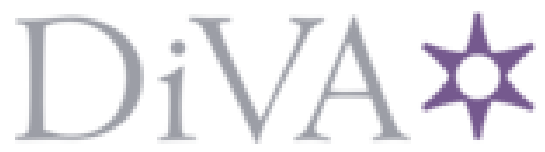

http://www.diva-portal.org

This is the published version of a paper presented at 2015 IEEE 28th International Symposium on Computer-Based Medical Systems.

Citation for the original published paper:

Kane, B., Luz, S. (2015)

Clinical Training and Teamwork: Learning and Feedback

In: 2015 IEEE 28th International Symposium on Computer-Based Medical Systems (pp. 280-285). IEEE

IEEE International Symposium on Computer-Based Medical Systems

https://doi.org/10.1109/CBMS.2015.79

N.B. When citing this work, cite the original published paper.

(C) 2019 IEEE. Personal use of this material is permitted. Permission from IEEE must be obtained for all other uses, in any current or future media, including reprinting/republishing this material for advertising or promotional purposes, creating new collective works, for resale or redistribution to servers or lists, or reuse of any copyrighted component of this work in other works.”

Permanent link to this version:

http://urn.kb.se/resolve?urn=urn:nbn:se:kau:diva-75168 


\section{Clinical Training and Teamwork: Learning and Feedback}

\author{
Bridget Kane \\ Centre for Health Policy and Management \\ Trinity College \\ Dublin, Ireland \\ kaneb@tcd.ie
}

\author{
Saturnino Luz \\ School of Computer Science and Statistics \\ Trinity College \\ Dublin, Ireland \\ luzs@acm.org
}

\begin{abstract}
MDTMs are now a feature of routine hospital work and provide a valuable learning opportunity for education and practice development. The popularity of the forum as a patient management mechanism has had a negative counter effect on the educational function of the forum. Behavioural interventions and technical supports are identified based on long term ethnographic studies to restore the educational benefits of the forum. The potential for re-developing the MDTM into a rich educational resource that will assist in clinical education, professional development, provide an evidence base for guideline development by integrating clinical outcome feedback into the meeting record is proposed.

Index Terms-clinical education; learning; knowledge development; Continuing Medical Education (CME); Computer Supported Cooperative Work (CSCW); Multidisciplinary Medical Team Meetings
\end{abstract}

\section{INTRODUCTION}

Multidisciplinary teams (MDTs) and their meetings (MDTMs) have become a feature of routine practice in modern hospitals, especially university teaching hospitals. An MDT comprises a group of specialists, (physicians, surgeons, oncologists, nurses, pathologists and radiologists), who are potentially involved in a patient's care. They meet and discuss each of their patients, one at a time; review all of the facts in the patient's case, and agree a diagnosis and treatment plan. Within MDTs each specialist contributes his, or her, particular skill and knowledge with respect to the patient's condition in discussion. Through the interaction of specialists at the MDTM, new knowledge is generated that is unavailable elsewhere, and its value as a forum for professional education, training and development, as well as organisational learning, has been demonstrated [1]. The patient case discussion (PCD) is described in detail in [2].

\section{A. The Educational Function of MDTMs}

The MDTM has its roots as a forum for clinical education and practice development. It has grown from its role in teaching undergraduate and postgraduate medical students and has its origins in clinical-pathology, clinical-radiology and surgical risk management meetings [3]. The MDTM has evolved as an important mechanism in patient management in response to the increasing complexities of patient care and demands for improved quality of services. It now serves several purposes, which encompass patient management, education, organisation and social functions. For example, it provides a mechanism for the introduction to new concepts and techniques in both clinical and experimental medical sciences; audit is facilitated and information is managed through MDTM activities. Its social function is significant in its contribution to staff morale and team-building.

MDTMs continue to serve educational functions for undergraduate medical students. The MDTM provides an opportunity to explore scientific background and recent medical literature pertaining to the area. Furthermore, the introduction of new concepts and techniques in specialist areas, such as pathology and radiology can be facilitated through these meetings. There is also an important opportunity to extend experience with unusual cases by sharpening diagnostic skills on cases that are uncommon, or that present specific diagnostic dilemmas. MDTMs are an accreditation requirement for postgraduate specialist programme and recognised as continuing professional development (CPD) /continuing medical education (CME) for clinical staff [4]. The services provided by MDTMs fulfil its roles and functions as a system to enhance patient care [5] and facilitate learning [6] for individual participants, and for the hospital.

From the hospital's learning perspective, the group engages in peer review, and the forum provides potential for ongoing learning and education. MDTMs provide clinicians with exposure to a range of clinical experience which would be difficult to acquire otherwise, such as cases that are uncommon, involve difficult decisions, or that present specific diagnostic dilemmas. From a knowledge management perspective, MDTMs serve quality process objectives and allows for comparison between different investigative procedures in the diagnostic task. Thus, in addition to its learning and patient management functions, the MDTM contributes information for the development of quality processes, including clinical practice guidelines, and can be regarded as an organisational quality mechanism [7].

Over recent years, University teaching hospitals have typically experienced an exponential increase in workload being processed through the MDT structures which has introduced pressure on the MDTM as a system serving multiple purposes [8]. 
We have found that as the importance of this forum in process quality improvement and patient management has become evident, and we note that the educational value has become more diminished. The education of under-graduates, postgraduates specialist education and training, clinical training for interns and undergraduate medical students, as well as professional development of medical and other staff, which was at one time the primary function of the meeting, has become a concurrent secondary function.

In this paper we report on observations that demonstrate the weakening of the educational function, and provide supporting evidence for this interpretation. We propose both behavioural and technical supports that can be expected to strengthen the educational value of this MDTM forum, though our study findings.

\section{Methodology}

A long term ethnmethodologically-informed ethnographic study was conducted with MDTs at a large teaching hospital, following standard methodology associated with fieldwork in workplace settings [9]. Observation findings were explored and validated in surveys, questionnaires and interviews with team members. For this study we re-examined data that were gathered at MDTMs, and in two surveys over a two year period early in our work, in the context of later knowledge gained later in the project. The surveys were conducted among observer participants during a series of case discussions for which case-related detailed data were gathered, namely vocal participation by radiologists, clinicians and pathologists [10] and the use of images during discussion. Observer participants include students, and allied health professionals who take notes to inform later patient care tasks and responsibilities.

One of the exercises we re-examined was conducted with observer participants attending MDTMs as part of their professional training. For each of a series of cases recorded we asked the observers to rate the educational value of the patient case discussion (PCD), during the meeting. The focus on the observer participants is justified in terms of the educational role of the MDTM [5].

The second exercise we re-examined concerned the perceived benefit of active participation in the patient case discussion (PCD). All participants were invited to respond to a questionnaire that enquired about the relative contribution and gain in information for their role. This second exercise was conducted independent of the meeting process.

Pearson's Chi-square $\chi$ tests were used to check for differences in categoric responses (correct, incorrect and nonresponses) where appropriate. Scaled variables such as the number of responses per participant were compared under different conditions using $t$ tests, and the Mann Whitney $\mathrm{U}$ test was used when comparing rating responses for the presentation of clinical findings and the educational rating.

\section{FIndings AND ANALYSIS}

The MDTM process involves much more than actual time spend at an MDT meeting. The preparation before the meeting, and the follow-up tasks afterwards are an integral part of the MDTM process.

\section{A. Pre-MDT Meeting}

Depending on the role involved, preparation for the meeting is different, and is influenced by the expectations of their role contribution to the MDTM, and the anticipated benefits from their participation in the PCD. We identified three types of pre-MDTM role category activities, as follows:

1) Roles who contribute patient findings to the MDTM

2) Roles who anticipate they may be treating a patient following the outcome of the meeting

3) Roles who are actively interested in learning new knowledge, sharpening their diagnostic skills or interested in learning about the newest diagnostic approaches.

1) For those roles who gather information before the MDTM with the intention of presenting salient findings, they report that they learn in the process. As one radiologist described:

It is one thing to give your diagnosis from the comfort of your office, but it is quite another thing to explain to the group how you came to your decision. It makes you think it through more carefully ...

Those roles who to present to the MDTM also say:

Preparation of MDTM material will keep potential teaching points in mind that can be shown at the meeting.

MDTM preparation also serves an education function within the respective departments for radiologists and pathologists in training before the actual MDTM. Non-Consultant Hospital Doctors (NCHDs) report that preparing to present the patients clinical history with important signs and symptoms is a challenge from which they learn how to recount the patient narrative succinctly to the MDTM.

2) Roles such as oncologists are interested in knowing if any of the patients due to be discussed have a history in their care. Their previous experience of managing a patient may significantly influence the decision on the best approach in managing the latest event. For example if a patient has had surgery in the past, and experienced a particularly difficult recovery period, a surgical intervention to the current problem may be unwise, if other options are available. Such a discussion will likely require additional information before a decision to perform surgery is considered.

3) Many of the roles present would like to be able to review cases afterwards, to learn how the decision was taken and about the information on which the decision was based. This need was raised on several occasions and prompted the development of a meeting record, reported in [11], [12], and systems of record-keeping have now been implemented.

However, now that records of the MDTM decision are available in the patients electronic chart (EPR), it is not so clear on enquiry if the treatment decision was implemented, by what date, and what was the final outcome for the patient. A new system requirement has emerged, namely to know what happened to the patient subsequent to the MDTM decision

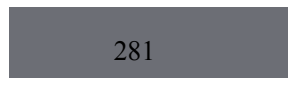


being made, and presumably implemented. While the EPR It would be good if the final outcome could be linked back to the MDTM decision, from learning, and audit, perspectives. Such a record would be a useful educational tool and facilitate research and clinical practice development.

\section{B. Learning at the MDTM}

The obvious learning opportunity during the MDMT is the individual patient cases that are presented. Those in attendance hear about the patient's presentation, radiology findings and pathology results. Observers then witness discussion on the findings and the agreed best next step in the patient's management. Efforts are on-going to enhance the MDTM and learning opportunities though providing easier assess to clinical practice guidelines and /or related cases. Having such tools to access relevant information would be valuable to the MDTM [13].

Consultant clinicians and clinical trainees, especially pathologists and radiologists, report that they regularly attend MDTMs even though they may not be directly involved in the case, as they find benefit from the discussion. Having more than one person from a speciality allows for enhanced peer review and more in-depth case discussion at MDTMs. All participants claim to learn from their attendance at MDTMs, and we demonstrate, illustrated in Figure 1, that those who are active contributors gain more, and the more actively they participate the greater their personal gain [14].

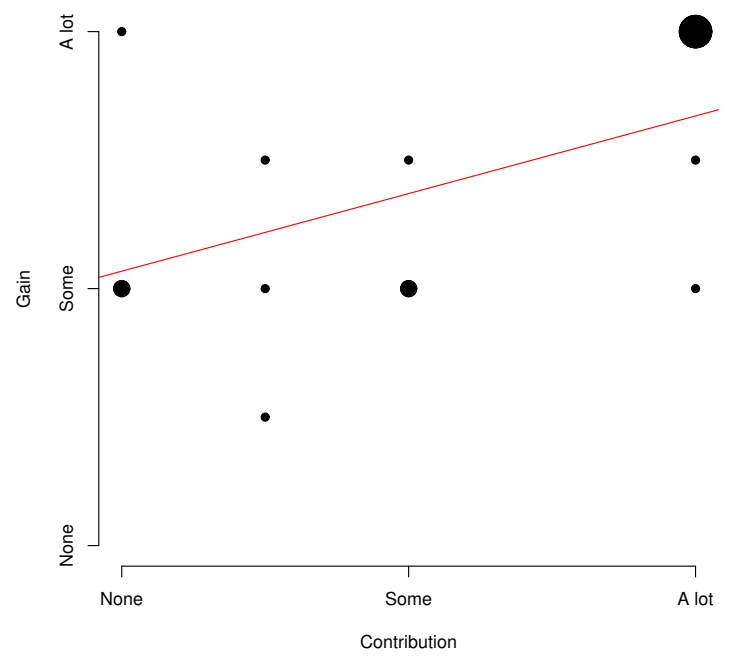

Fig. 1. Contribution versus Gain

The case discussion at an MDTM can be likened to a problem-based learning (PBL) exercise as described by [15], where the case presentation in the small group is a stimulus to learning. In the case review, with the patient at the centre of the team's focus, there is opportunity to integrate theory with clinical components and the activity demands knowledge acquisition. An important part of the PBL approach however, is the ability for the participants to recognise their success and their achievement in making the best decision. This is not currently possible within the current system of record-keeping and we focus on this later in our discussion.

\section{Post-MDT Meeting}

Currently there is an MDTM decision record in the patient's EPR. There is a separate record maintained of the list of patients discussed, and the attendees at each MDTM. A weakness is identified in the lack of direct feedback mechanism to the MDTM that the decision agreed has been acted upon, and the clinical outcome of the decision is not easily available.

In earlier times, when the primary function of the MDTM was its educational role, patient cases would be identified for discussion, once all their treatment was completed. These cases were carefully compiled and presented and there was an in-depth discussion among the senior and junior staff. Typically, senior staff would question the students on their opinion about their anticipated outcome. For example, the clinician might ask: This middle aged woman presented to the $A \& E$ with difficulty in breathing, what would you have done? What investigations would you have requested? The students were questioned, and they would each express their opinion before the next part of the story would be revealed, such as: There was a mass in the RUL on chest $x$-ray. What would be the next investigations you would carry out?

The discussion would continue in this manner with several tangents explored using the counterfactual: But what if ... were the situation?. The final outcome, whether the patient improved and was discharged, or died, or evolved into a chronic condition, would eventually be revealed. More senior students were expected to write up these 'case studies' for publication in local journals, as part of their training.

Having such detailed discussion enhanced learning for those present. As well as the clinical presentation and natural history of disease processes, students learned an appreciation for the information needs of others in the MDT. For interpretative tasks in radiology and pathology, for example, contextual information is important for accurate interpretation. Lack of information is a potential source of medical error, and often not provided by front-line clinical staff. Through rich interaction during a case discussion, clinical staff gain a greater understanding of the importance of providing appropriate information when requesting pathology (or radiology) in the future. This potential lack of understanding of each other's professional needs was recognised in Symon's study of a radiology department [16]. MDTMs provide a useful opportunity for mutual education of requirements for the conduct of individual task activities.

\section{Rating the Educational Value of the PCDs}

In the survey rating the educational value of PCDs, participants were asked to submit a score for the clarity of clinical, radiological and pathology findings and the educational value of the discussion. As one might predict the educational value 
correlates positively with the quality of the verbal clinical presentation, and with the quality of the pathology and radiology images. The evaluation of the educational benefit reported correlates highly with the clarity of the clinical presentation $(p \leq 0.01)$. The study is reported more fully in [17] but it is important to note here the value of co-located discussions that are relatively less structured than those held in videoconference, reported below.

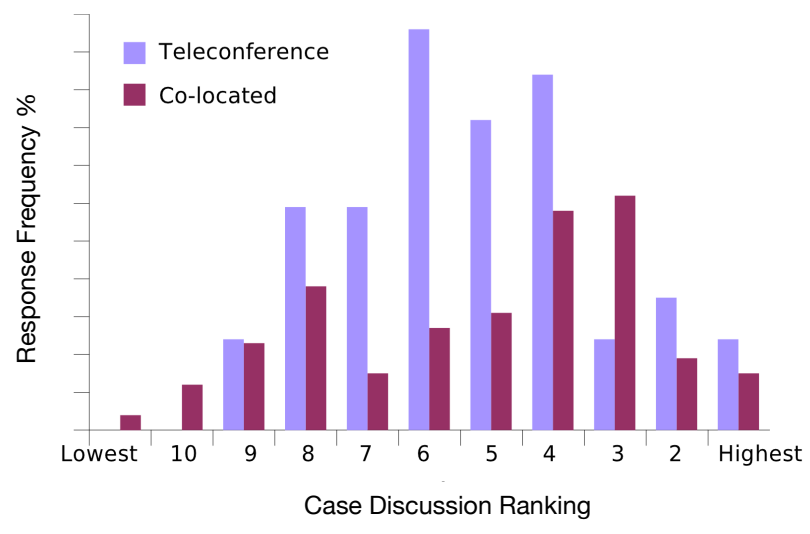

Fig. 2. Educational Value

1) PCDs in Videoconference: PCDs in videoconference are characterised as i) taking more time, ii) fewer participants, iii) no NCHD participation, iv) clearer audio.

It was anticipated that the increased time it takes to hold a PCD in videoconference, and the fact that cases selected for discussion in videoconference are typically complex, that the educational value for observers would be enhanced. Contrary to expectations, and shown in Figure 2, while videoconference PCDs generally scored well, it was found that the cases were rated as having less educational value than expected. On the other hand, ratings for co-located PCDs seem to follow a bimodal distribution. Observation and interview data inform us that the formality in videoconference provides a protocol that improves the weakest discussions, but that this formality also inhibits questioning and argument. Observers welcome more informal, relaxed, co-located discussions where senior staff argue their viewpoint and exchange frank opinion on cases. Thus some co-located discussions can be more 'animated'; and observers consider that they learn more when experienced staff articulate their opinion and doubts on a case, and there is more interaction in the discussion.

When videoconferencing was first introduced, it was not possible to see remote participants while reviewing radiology or pathology images, due to the configuration of audio and video streams. The Mean frequency distribution (\%) for the clarity of clinical findings in PCDs in the co-located setting was higher than for PCDs in videoconference, which is consistent with Bly's observation regarding the need to see things while talking about them [18]. This fact may have impacted on the perceived educational value of PCDs in videoconference.

Where enough time is taken, participation rate is high, all the information is available in the discussion, and all the necessary roles are present, PCDs tend to achieve a high score with respect to their educational value. Observers particularly like when there is some argument, or discussion among the senior experts as to some fine detail in the case. For example, the clinicians might question the radiologist as to why a needle biopsy is not feasible, and the radiologist explains about the difficulty in accessing the lesion because of its location. A mass that is close behind a rib, or adjacent to the aorta may render the procedure too difficult or too risky to undertake. Pathologists may not be able to conduct particular tests because the biopsy was preserved in formaldehyde, and a discussion might take place as to how best to differentiate, or classify, a tumour (which will influence the treatment modality to be adopted).

While PCDs in videoconference may 'satisfy' overall, there were differences experienced between those participants at the main centre, and those at the smaller remote hospital. Figure 2 was generated from the evaluations by participants at the large teaching hospital. Interviews and observation at remote sites determined that the technical quality was less than satisfactory but the possibility of connecting to the large centre is highly valued. Remote participants typically said: We value the connection, even though the quality is poor - but if you think this is bad, it used to be much worse! We suffer the shortcomings because we really want to keep in touch with [...].

Having access via videoconference to learn about complex cases that are being managed at the large centre is highly valued by remote hospitals. When videoconferencing was first introduced, it was common for the remote participants to observe PCDs at the large centre, for education purposes.

$51 \%$ of responses in videoconference and $63 \%$ of colocated discussions were rated in the top half of the perceived 'educational value' scale. This apparent preference for PCDs in co-located settings is reflected in the results of other evaluations of videoconference [19], [20]. Reporting high levels of satisfaction for many attendees, Fielding et al [21] noted comments that face-to-face meetings are preferred and those with less satisfaction scores do not always attend meetings.

\section{E. Recent Developments and Future Trends}

Recent studies have shown that TIME is the enemy of the MDTM as a forum for clinical education and professional development [22], [8]. The popularity of the MDTM as a forum for patient management has resulted in a dramatic increase in the number of patients being processed through the MDTM structure. For some MDTs increases of over $200 \%$ were recorded [22]. The increase in the number of PCDs being discussed per hour has had the impact of fewer participants involved in the discussion, active participation being undertaken by more senior roles, a reduction in the amount of imaging being shown and fewer attendees. MDTMs have been recently described as "getting down to business" and that "Some people leave once their patient has been discussed". Staff continually complain that there is "not enough time" devoted to MDTMs, 
and that the education / teaching element of the MDTM is suffering as a result. The situation remains unresolved.

An initiative in Aberdeen where live MDTMs were videolinked to a teaching forum with a dedicated tutor was found to be successful [23]. Having a safe learning environment, related to, but separate from, the main MDT was found useful. The pace of delivery in the teaching setting is slower than in the main MDTM and better suited for students, and the language used by consultants at the MDTM was broken down 'to plain English' in the video-linked student-led teaching setting [23].

As well as the MDTM providing an important opportunity for clinical training and education, several studies have demonstrated that educational initiatives aimed at team training can improve the team's performance, and has a direct positive impact on patient clinical outcomes [24]. Indeed, it is suggested from studies of distance education [25] that improving team cohesiveness will likely improve the educational value of discussions in videoconference. We can interpret from these studies that the value of the MDTM as an educational forum can be further enhanced though training the MDT and enhancing its cohesiveness.

\section{DISCUSSION}

The MDT is a 'community of practice' and learning is a characteristic of such communities [26]. Thus MDTMs, where members interact and collaborate, can be expected to provide a valuable opportunity for education and professional development. In this example of MDTMs, group knowledge is achieved through group discourse, is spread across people and artefacts and persists in physical artefacts. Verbal articulation and dialogue is recognised to have considerable promise as a problem-formulation and problem-solving philosophy and technology [27] as well as learning benefits [28]. The articulation of thought, and connecting new experiences to existing knowledge, enhances the development of clinical reasoning [29], [30]. Communicating or justifying one's views is shown to yield cognitive benefit [31]. People learn through dialogue with one another and transform their understanding in the process [28].

Apart from the educational benefit of dialogue, the development of dialogue among different specialities is critical for good communication and organisational effectiveness. Hospitals are increasingly dependent on valid communication, which increasingly hinges on the development of a common language and any form of organisational learning requires the evolution of shared mental models. Dialogue is a necessary first step in the development of those shared mental models [27].

In this paper, the term 'information' is used mainly to refer to patient data items and disease processes. There are other levels of information processing that are evident at MDTMs, namely the internalisation and feedback that is embedded in the exchanges at individual, group and at hospital organisation level that should be noted as part of the education and learning that takes place at MDTMs. The education activities to be supported need to be considered from the perspective of an individual team member, the interactions that occur between team members and with the knowledge-capture need for the organisation, i.e. education solutions should be sought at the individual, group and organisation levels.

\section{A. Missing Pieces}

In our study and discussion of MDT work, we have tended to look toward a meeting record to fulfil the need for an educational resource (as well as a patient record). However further analysis reveals that the missing part is in fact FEEDBACK. Currently there is no easy way of determining if i) the decision agreed was acted upon, or ii) the clinical outcome for the patient. If a record can be generated that would capture the subsequent treatment / intervention activity and clinical outcome we would have a valuable repository of patient cases that are complete in the information available and used to make decisions, as well as the interventions and clinical outcomes of the decision. Such a repository of cases could also be used as a resource for audit, and evidence base for practice development and practice reflection.

\section{CONCLUSION AND RECOMMENDATIONS}

Both behavioural interventions and technical support would enhance the MDTM as an educational forum, as well as a place where patient management is conducted safely, and teamwork is reinforced.

1) Participation: The ideal case discussion at an MDTM would have high levels of participation by team members, questioning and discussion. Such interactions would also help guard against the development of 'Groupthink' and promote more 'teamthink' [32]. Active vocal participation in patient case discussions reinforces learning and facilitates the acquisition of new knowledge. From our study of information gain during PCDs, we demonstrate that the vocal articulation of patient findings when making contributions to the patient case discussion benefits the provider of the information, as well as being of benefit the addressees.

2) Use of Images: Aside from a good work practice, but from a learning perspective, it is imperative that clinicians have access to see the patient's radiology and pathology images during a discussion (PCD) on the case, irrespective of whether or not the discussion is held in videoconference. Being able to review the image detail is a critical part of the peer review, is essential for the understanding and learning that takes place during discussion, and helps in achieving common ground.

3) Space and Time: In the ideal meeting room, all participants would have the ability to connect via wireless connection to the data displayed on screen, save a copy on their personal device, to annotate and/or take personal notes. Personal notes could be used for reference purposes in post-MDTM tasks and responsibilities and also support educational and research interests, using a tool such as developed with this project [33]. In the ideal setting there would be adequate time incorporated into the weekly schedule to accommodate comprehensive case discussion at MDTMs. 
4) Feedback: Feedback is the heart of medical education, and one of the basic teaching methods used in clinical settings [34]. Although most clinicians are familiar with the principles of giving feedback, many clinicians probably do not recognise the many opportunities presented to them for using feedback as a teaching tool [34], such as in MDT settings.

Feedback to the MDT on the clinical outcome of the case would complete the communication - feedback loop and can be predicted to improve work practices in the long term. Such feedback could potentially be provided through technical support that would include a mechanism to link the PCD record to subsequent clinical interventions, and clinical outcomes and improve patient safety.

Having a PCD record with subsequent clinical outcomes linked to it, would provide a valuable repository of for clinical education and training, professional development and improve teamwork.

\section{Acknowledgment}

We wish to acknowledge the generous participation of staff at St. James's Hospital Dublin in this work.

\section{REFERENCES}

[1] E. Oborn and S. Dawson, "Learning across communities of practice: An examination of multidisciplinary work," British Journal of Management, vol. 21, no. 4, pp. 843-858, 2010.

[2] B. Kane and S. Luz, "Achieving diagnosis by consensus," Computer Supported Cooperative Work (CSCW), vol. 18, no. 4, pp. 357-391, 2009.

[3] K. Wong and D. Birks, "Surgical risk management: the value of a weekly surgical radio-pathological meeting," Anz J Surg, vol. 74, no. 4, pp. 205209, April 2004.

[4] The Irish Medical Council, "Continuing medical education/ continuing professional development," 2007.

[5] E. A. Newman, A. B. Guest, M. A. Helvie, M. A. Roubidoux, A. E. Chang, C. G. Kleer, K. M. Diehl, V. M. Cimmino, L. Pierce, D. Hayes, L. A. Newman, and M. S. Sabel, "Changes in surgical management resulting from case review at a breast cancer multidisciplinary tumor board," Cancer, vol. 107, no. 10, pp. 2346-2351, 2006

[6] M. Jefford, R. Jennens, T. Speer, and V. Thursfield, "Different professionals' knowledge and perceptions of the management of people with pancreatic cancer." Asia-Pacific Journal of Clinical Oncology, vol. 3, pp. 11-51, 2007.

[7] A. Fleissig, V. Jenkins, S. Catt, and L. Fallowfield, "Multidisciplinary teams in cancer care: are they effective in the UK?" Lancet Oncology, vol. 7, pp. 935-943, November 2006.

[8] B. Kane and K. Groth, "Multidisciplinary work practices: A comparison of three major European hospitals," in IEEE 27th International Symposium on Computer-Based Medical Systems. IEEE, 2014, Conference Proceedings.

[9] D. Randall, R. Harper, and M. Rouncefield, Fieldwork for Design. Springer, 2007.

[10] S. Luz, "The non-verbal structure of patient case discussions in multidisciplinary medical team meetings," ACM Transactions on Information Systems, vol. 30, no. 3, pp. 17:1-17:24, 2012.

[11] B. Kane, P. J. Toussaint, and S. Luz, "Shared decision making needs a communication record," in $C S C W$. ACM, 2013, pp. $79-89$.

[12] B. Kane and S. Luz, "On record-keeping at multidisciplinary team meetings," in 24th IEEE International Symposium on Computer-Based Medical Systems (CBMS). Bristol: IEEE, 2011.

[13] O. Frykholm and K. Groth, "References to personal experiences and scientific evidence during medical multi-disciplinary team meetings," Behaviour \& Information Technology, vol. 30, no. 4, pp. 455-466, 2011.

[14] B. Kane and S. Luz, "Information sharing at multidisciplinary medical team meetings," Group Decision and Negotiation, vol. 20, pp. 437-464, 2011.
[15] P. Hall and L. Weaver, "Interdisciplinary education and teamwork: a long and winding road," Medical Education, vol. 35, no. 9, pp. 867875, 2001.

[16] G. Symon, K. Long, and J. Ellis, "The co-ordination of work activities: Co-operation and conflict in a hospital context," Computer Supported Co-operative Work (CSCW), vol. 5, no. 1, pp. 1-31, 1996.

[17] B. Kane, S. Luz, and P. Toussaint, "Developing a framework for evaluation of technology use at multidisciplinary meetings in healthcare," in 26th IEEE International Symposium on Computer Based Medical Systems (CBMS). IEEE, 2013, Conference Proceedings.

[18] S. A. Bly, S. R. Harrison, and S. Irwin, "Media spaces: Bringing people together in a video, audio, and computing environment," Communications of the ACM, vol. 36, no. 1, pp. 28-46, 1993.

[19] G. Delaney, S. Jacob, R. Iedema, M. Winters, and M. Barton, "Comparison of face-to-face and videoconferenced multidisciplinary clinical meetings," Australasian Radiology, vol. 48, no. 4, pp. 487-492, 2004.

[20] E. J. Macaskill, S. Thrush, E. M. Walker, and J. M. Dixon, "Surgeons' views on multi-disciplinary breast meetings," European Journal of Cancer, vol. 42, pp. 905-908, 2006.

[21] R. G. Fielding et al., "Attitudes of breast cancer professionals to conventional and telemedicine-delivered multidisciplinary breast meetings." Journal of Telemedicine and Telecare, vol. 11, no. Supplement 2, pp. S2:29-34, 2005.

[22] B. Kane and S. Luz, “"'Do no harm”: Fortifying MDT collaboration in changing technological times," International Journal of Medical Informatics, vol. 82, no. 7, pp. 613-625, Jul. 2013.

[23] I. M. Thomas and J. L. Duncan, "Improving cancer teaching through videoconferenced multidisciplinary team meetings," Medical Education, vol. 47, no. 11, pp. 1133-1134, 2013. [Online]. Available: http://dx.doi.org/10.1111/medu.12346

[24] J. C. Morey, R. Simon, G. D. Jay, R. L. Wears, M. Salisbury, K. A. Dukes, and S. D. Berns, "Error reduction and performance improvement in the emergency department through formal teamwork training: Evaluation results of the medteams project," Health Services Research, vol. 37, no. 6, pp. 1553-1581, 2002.

[25] E. A. Williams, R. Duray, and V. Reddy, "Teamwork orientation, group cohesiveness, and student learning: A study of the use of teams in online distance education," Journal of Management Education, vol. 30, no. 4, pp. 592-616, 2006. [Online]. Available: http://jme.sagepub.com/content/30/4/592.abstract

[26] E. C. Wenger and W. M. Snyder, "Communities of practice: The organizational frontier," Harvard Business Review, vol. 78, no. 1, pp. 139-145, Jan/Feb 2000.

[27] E. H. Schein, "On dialogue, culture, and organizational learning," Reflections, vol. 4, no. 4, pp. 27-38, 2003.

[28] A. Collins, "Design issues for learning environments," in International perspectives on the psychological foundations of technology-based learning environments. Hilsdale, NJ: Lawrence Erlbaum, 1996, pp. $347-361$.

[29] J. I. Murphy, "Using focused reflection and articulation to promote clinical reasoning," Nursing Education Perspectives, vol. 25, no. 5, pp. 226-231, 2004.

[30] T. Koschmann, "Dewey's contribution to the foundations of cscl research." in Computer Support for Collaborative Learning (CSCL). Boulder, Colorado, USA: ICLS, 2002.

[31] R. J. Pingree, "How messages affect their senders: A more general model of message effects and implications for deliberation," Communication Theory, vol. 17, pp. 439-461, 2007.

[32] C. P. Neck and C. C. Manz, "From groupthink to teamthink: Toward the creation of constructive thought patterns in self-managing work teams," Human Relations, vol. 47, no. 8, pp. 929-952, 1994.

[33] S. Cameirano, B. Kane, and S. Luz, "Demonstration: Mobile annotation and recording at multidisciplinary medical team meetings," in ECSCW 2007. Limerick: ACM, 2007.

[34] W. T. J. Branch and A. Paranjape, "Feedback and reflection: Teaching methods for clinical settings," Academic Medicine, vol. 77, no. 12, Part 1, pp. 1185-1188, 2002. 